\title{
Hyperostosis in siblings
}

\author{
J W Spranger, ${ }^{1,2} \mathrm{MD} ; \mathbf{E}$ Lausch, ${ }^{2} \mathrm{MD}$ \\ ${ }^{1}$ Department of Endocrinology and Metabolic Diseases, Charité-Universitätsmedizin, Berlin, Germany \\ ${ }^{2}$ Division of Medical Genetics, Children's Hospital, University of Freiburg, Germany
}

Corresponding author: J Spranger (J.A.Spranger@gmx.de)

Infantile cortical hyperostosis - Caffey-Silverman disease - is a familial disorder manifesting in the late fetal period or infancy with excessive periosteal bone formation. Signs and symptoms regress spontaneously within months and result in expanded, deformed bones. The paucity of clinical symptoms may lead to delayed investigation and confusion of the remaining bone changes with those in other conditions. This problem is exemplified by two siblings misdiagnosed as osteogenesis imperfecta. The diagnosis of Caffey-Silverman disease was confirmed by molecular analysis showing the specific COL1A1 mutation in the patients and their clinically unaffected mother. Reduced penetrance rather than autosomal recessive inheritance explains multiple affected siblings born to healthy parents.

S Afr Med J 2016;106(6 Suppl 1):S98-S99. DOI:10.7196/SAMJ.2016.v106i6.11007

In 1930, the German paediatrician Roske ${ }^{[1]}$ described an infant with 'a peculiar bone disease' that today would be diagnosed as infantile cortical hyperostosis (ICS). This term was introduced in 1945 by Caffey and Silverman, who more precisely defined the disorder and whose names are often attached to it. ${ }^{[2]}$ ICS usually manifests during the first 6 months of life with irritability, fever, anorexia, and painful, firm, soft-tissue swelling, particularly at the jaw and extremities. Laboratory studies occasionally reveal moderately elevated alkaline phosphatase levels and prenatal occurrence has been observed. ${ }^{[3,4]}$ Severely affected patients may die during infancy; others survive after a protracted course and develop bowing and/or increased length of the involved long bones. Early radiographs show cortical hyperostosis of the affected sites. In protracted cases, the original cortex is resorbed, while the medullar canal widens with bowing and longitudinal overgrowth.

The disorder is caused by a recurrent heterozygous mutation of the COL1A1 gene (c.3040C $>T)$ leading to a substitution of arginine by cysteine at position 1014 (p.Arg1014Cys, formerly described as p.Arg836Cys) of type 1 collagen ${ }^{[5]}$ Autosomal dominant inheritance has been proposed in Roske's original patient, who fathered a similarly affected child. ${ }^{[6]}$ Autosomal recessive inheritance has also been claimed. ${ }^{[7,8]}$

Here, we describe sisters with cortical hyperostosis manifesting with bowing deformities of the long tubular bones. Bone changes and birth to seemingly unaffected parents raised the suspicion of autosomal recessive osteogenesis imperfecta.

\section{Case reports}

Patient 1 was born to distantly related, healthy parents after an uneventful pregnancy, weighing $2.6 \mathrm{~kg}$ at birth. Down syndrome was suspected on clinical grounds and confirmed by chromosome analysis showing a $t(14 ; 21)$ translocation. Swelling of the right tibia, and of the left tibia 3 months later, was recorded. Radiographs disclosed bowed and widened tibiae, fibulae and left radius and were interpreted as a metaphyseal dysplasia or osteogenesis imperfecta. Clinical examination at 2 years of age showed an alert child with a flat face, upslanting palpebral fissures, single palmar crease, muscular hypotonia, marked motor delay and other distinct features of Down syndrome but with no cardiac defect. Anterior bowing of the tibiae was noted. Body height was $90 \mathrm{~cm}$ (85th percentile). Routine and bone-specific laboratory values were normal including serum $\mathrm{Ca}, \mathrm{PO}_{4}, \mathrm{AP}$, urinary pyridinoline and deoxypyridinoline. Radiographs showed wide and bowed shafts of both tibiae, fibulae, ulnae, radii and the left femur (Fig. 1A).
Patient 2, the younger sister of patient 1 , was born with a weight of $2.8 \mathrm{~kg}$ after an uneventful 40 -week gestation. At 3 months of age, painless swelling of her left forearm was noted. Examination at 1 year showed a well-developed girl with a normal height of $79 \mathrm{~cm}$ and normal psychomotor development. A mildly bowed left forearm was the only abnormality. Routine and bone-specific laboratory values were normal. Radiographs showed mild bowing of the left radius and a mild deformity of the left radius (Fig 1B). Other sites were normal Figs $2 \mathrm{C}$ and $2 \mathrm{D}$ ). On account of the family history, a diagnosis of

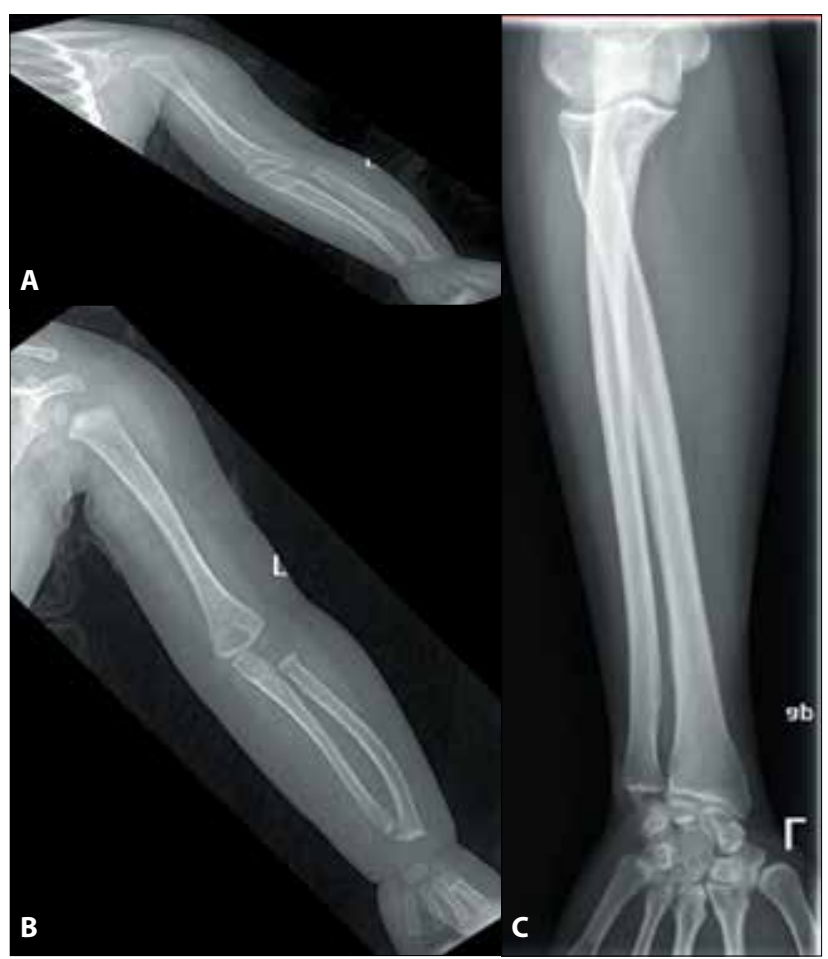

Fig 1. (A) Patient 1 at 2 years old. The diaphyses of the arm bones are irregularly widened with mild ulnar bowing of radius and ulna. The cortices are dense and smooth. Bone structure is flawed. (B) Patient 2 at 1 year old. The bone changes are similar to those in the older sister but less pronounced. The cortices appear normal. (C) Mother of patients 1 and 2. Normal bones. 


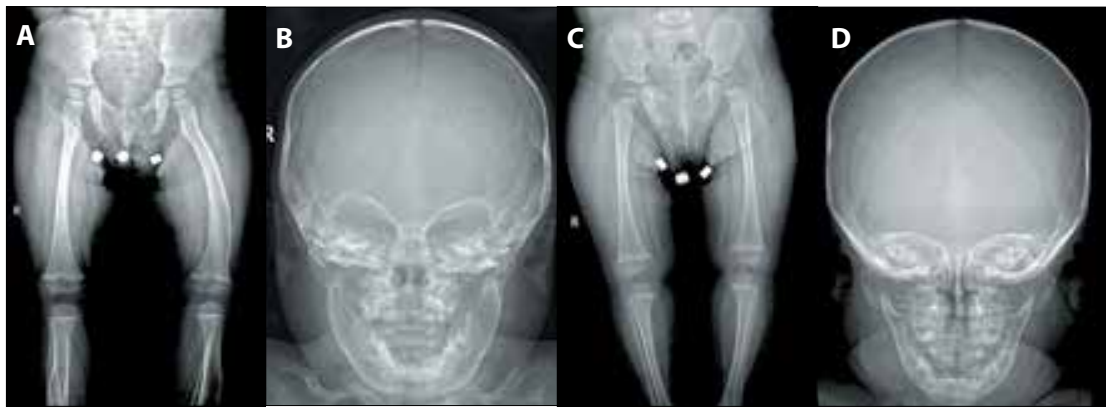

Fig. 2. (A) Patient 1 at 2 years old. The diaphyses of the left femur, both tibiae and fibulae are expanded and bowed; the cortices are smooth and thin; (B) The mandible is wide with normal cortices. (C) and (D) Patient 2 at 1 year old. Normal bones.

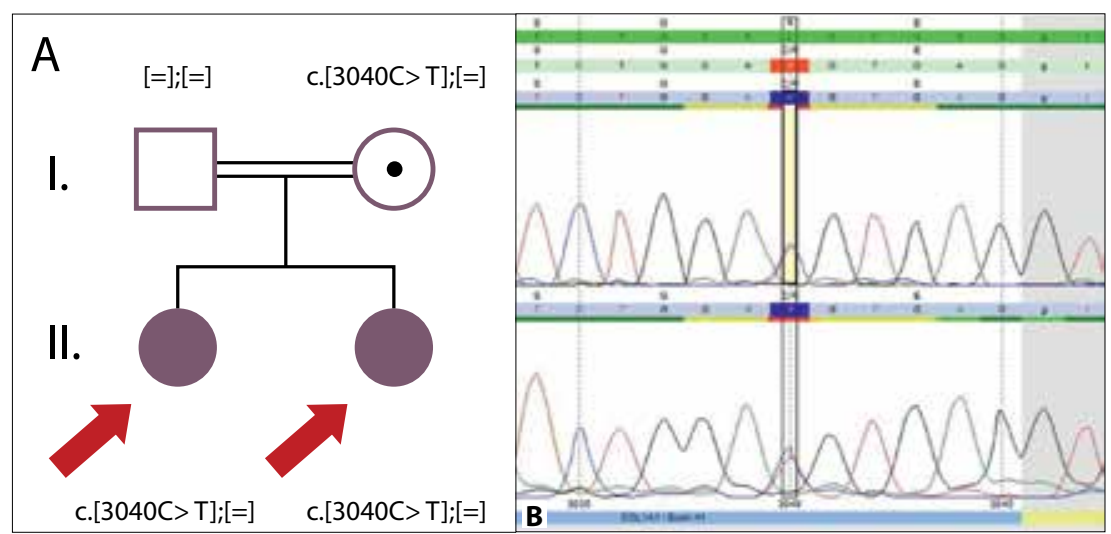

Fig. 3. (A) Abridged pedigree of the family. (B) Representative capillary electropherogram of exon 41 of the COL1A1 gene. The sisters and their mother carry the recurrent heterozygous c.3040C $>\mathrm{T}$, p.Arg1014Cys mutation associated with autosomal dominant Caffey-Silverman disease.

autosomal recessive osteogenesis imperfecta was considered.

\section{Molecular analysis}

DNA was extracted from peripheral blood by standard protocols; Sanger sequencing analysis of exon 41 of the COL1A1 gene demonstrated a heterozygous c.3040C $>T$, p.Arg1041Cys transition in both girls. The same sequence change was also detected in the clinically inconspicuous mother; the father carried no mutation (Fig. 3).

\section{Discussion}

Periosteal thickening and detachment from underlying bone were illustrated in the original article by Roske ${ }^{[1]}$ (Fig. 4) and remain the radiographic hallmarks of Caffey-Silverman disease. With regression, the excessive bone is resorbed and the affected long bones are remodelled leading to bowing and medullary distention of the long tubular bone shafts, mandibles and other bones. If, as in our cases, this state has already been reached at the time of investigation, other causes of bowing and diaphyseal expansion may enter the differential diagnosis, such as osteogenesis imperfecta, hypophosphatasia, and ill-defined forms of congenital bowing. ${ }^{[10-12]}$ The lesson to be learned is that absence of distinct periosteal hyperostosis does not rule out CaffeySilverman infantile cortical hyperostosis.

Mild to moderate bone changes in the siblings and their absence in the molecularly affected mother demonstrate the variable expression of the disorder. This has also been shown in large kindreds with vertical transmission of Caffey-Silverman disease. ${ }^{[12-15]}$ The heterozygous COL1A1 mutation in the clinically unaffected mother of our patients suggests that reduced penetrance and not autosomal recessive inheritance explains the recurrence of the disorder in affected siblings of seemingly unaffected parents. Adequate genetic counselling requires molecular analysis of both parents of an affected child.

\section{References}

1. Roske G. Eine eigenartige Knochenerkrankung im Säuglingsalter. Mschr Kinderheilk 1930;47:385-400.

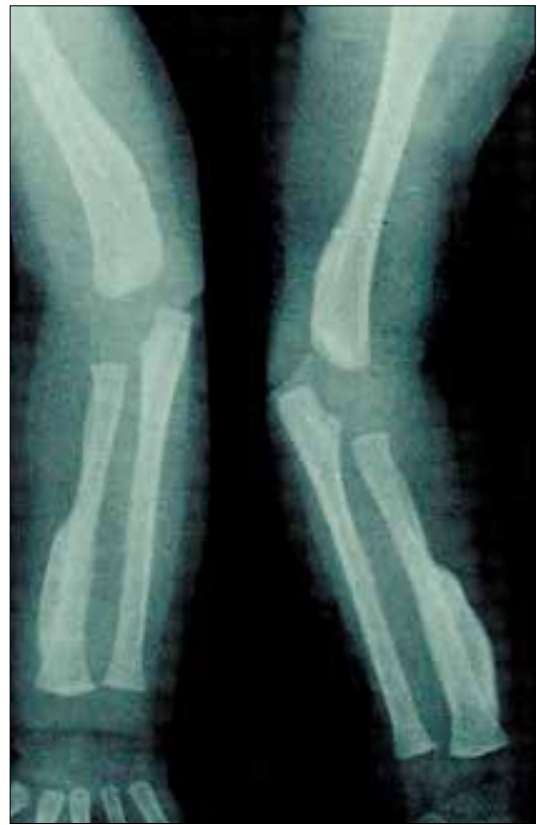

Fig. 4. Reproduction of Fig. 5 from the original description of infantile cortical hyperostosis by Roske, ${ }^{[I]}$ showing the characteristic cortical hyperostosis in the lower segments of humeri and radii.

2. Caffey J, Silverman W. Infantile cortical hyperostosis Preliminary report on new syndrome. Am J Roentgenol Radium Ther 1945;54:1-16.

3. Nemec SF, Rimoin DL, Lachman RS. Radiological aspect of prenatal-onset cortical hyperostosis (Caffey Disease). Europ J Radiol 2012;81(4):e565-e572. DOI:10.1016/j. ejrad.2011.06.049

4. Schweiger S, Chaoui R, Tennstedt C, et al. Antenatal onset of cortical hyperostosis (Caffey disease) case report and review. Am J Med Genet 2003;120A(4):547-552. DOI:10.1002/ ajmg.a.20062

5. Gensure RG, Määkitie O, Barclay C, et al. A novel COL1A1 mutation in infantile cortical hyerostosis (Caffey disease) expands the spectrum of collagen-related disorders. J Clin Invest expands the spectrum of collagen-related disor

6. Valler K, Laur A. Zur Ätiologie der infantilen kortikalen Hyperostose (Caffey Syndrom). Fortschr Geb Röntgenst 1953;79(4):446-452. DOI:10.1055/s-0029-1232243

7. Barba WP, Freriks DJ. The familial occurrence of infantile cortical hyperostosis in utero. J Pediat 1953;42(2):141-150. DOI:10.1016/s0022-3476(53)80207-6

8. Sidbury JB Jr, Sidbury JB. Infantile cortical hyperostosis. An inquiry into the etiology and pathogenesis. N Engl J Med 1954;250(8):309-314. DOI:10.1056/nejm 195402252500802

9. Le Merrer M, Cormier Daire V, Maroteaux P. Re-evaluation of kyphomelic dysplasia. Am J Med Genet 2003;120A(2):289-291. DOI:10.1002/ajmg.a.20035

10. Cisarik F, Kozlowski K, Masel J, Sillence D. Variability in kyphomelic dyplasia. Pediatr Radiol 1999;29(7):551-557. DOI:10.1007/s002470050644

11. Hall BD, Spranger J. Congenital bowing of the long bones. A review and phenotype analysis of 13 undiagnosed cases. Eur Pediatr 1980;133(2):131-138. DOI:10.1007/bfo044158

12. Gerrard JW, Holman GH Gorman AA, Morrow IH. Familial infantile cortical hyperostosis. J Pediatr 1961;59(4):544-548 DOI:10.1016/s0022-3476(61)80238-2

13. Ceretti-Mainardi P, Venturi G, Spunton M, et al. Infantile cortical hyperostosis and COL1A1 mutation in four generations. Eur J Pediatr 2011;170(11):1385-1390. DOI:10.1007/s00431011-1463-0

14. Suphapeetiporn K, Tongkobpetch S, Mahayosnond A Shotelersuk V. Expanding the phenotypic spectrum of Caffey disease. Clin Genet 2007;71(3):280-284. DOI:10.1111/j.1399disease. Clin Gent

15. Newberg AH, Tampas JP. Familial infantile cortical hyperostosis: An update. AM J Roentgenol 1981;137(1):93-96. DOI:10.2214/ajr.137.1.93 